\title{
Investigations on response time of magnetorheological elastomer isolator for real-time control implementation
}

\author{
Xiaoyu Gu, Yancheng Li and Jianchun Li \\ School of Civil and Environmental Engineering, Faculty of Engineering and Information Technology, \\ University of Technology Sydney, NSW 2007, Australia \\ * Corresponding authors: Jianchun.li@uts.edu.au; Yancheng.li@uts.edu.au;
}

\begin{abstract}
Utilizing the unique feature of MRE materials for vibration isolators has been intensively studied over the last several years. Real-time control of the MRE isolators holds the key to unlock MRE material's unique characteristics, i.e. instantly changeable shear modulus in continuous and reverse fashion. However, one of the critical issues for the applications of real-time control is the response time delay of MRE vibration isolators, which has not yet been fully addressed and studied. This paper identified the inherent response time of the MRE isolator and explored two feasible approaches to minimize the response time delay. Experiments were designed and conducted to evaluate the effectiveness of the proposed approaches on minimizing time delay on: i) the transient response of current of a large coil that generates magnetic field and ii) the transient response of shear force from the MRE isolator. The results show that the proposed approaches are effective and promising. For example, the proposed approach is able to reduce the force response time from $421 \mathrm{~ms}$ to $52 \mathrm{~ms}$ at rising and from $402 \mathrm{~ms}$ to $48 \mathrm{~ms}$ falling edges, respectively. Such level of short response time of the MRE isolators demonstrates the feasibility of application of real-time control and hence is the essential step on the realization of real-time control of vibration suppression system based on MRE isolator.
\end{abstract}

Keywords: Response time, Magnetorheological elastomer, Vibration isolator, Real-time control;

\section{Introduction}


Over the past two decades, magnetorheological elastomer (MRE) has gained considerable attentions in many engineering applications [1-6]. Being a solid analogy of magnetorheological fluid (MRF), MRE demonstrated its superiority over MRF for its fast response time, no sedimentation and sealing issues, etc. As such, MRE has been used to develop vibration isolation devices as well as actuators for various applications. Liao et al. [3] proposed a MRE vibration isolator with tunable stiffness and damping features. Du et al. [4] designed a seat suspension isolator and proposed an $\mathrm{H} \infty$ controller to minimize the driver body acceleration responses under different road conditions. Behrooz et al. [5] reported a variable stiffness and damping isolator (VSDI) for vibration mitigation of structures. Shi et al. [6] devised a vertical MRE isolator and investigated its dynamic behaviors when applied various current excitations. In civil engineering applications, $\mathrm{Li}$ et al [7] proposed a multilayer MRE base isolator for seismic protection of civil structures with more than 16 times increase in the stiffness. It has be noted that there is primary difference between applications of MRE and MRF, that is, MRE devices are usually designed as stiffnessvariable devices while MRF devices are predominantly designed for variable damping devices. Both types of devices are great candidates for real-time control applications.

For implementations of real-time control, a prompt response time of the control devices is critically important as the control command needs to be transferred to a control action instantly[8,9]. Consequences of undesirable response time, or time delay, may lead to a compromised control performance or even instability of the system $[10,11]$. There have been handful investigations on the response time issues of MRF devices, mostly on MR dampers [12-15]. For example, the typical response time of MR damper products from Lord Corporation, such as RD-1005-3, is around 20ms [14]. The well-known 180kN MR damper fabricated by Lord Corporation has response time of less than 60ms [14] which is considered to be adequate for structural control application in civil engineering structures [16]. It is 
commonly known that the main source causes time delay in MR dampers is not the response time of MR fluids (usually less than $1 \mathrm{~ms}$ ) but the inductance of the electromagnetics and the output impedance of the driving electronics [14].It is worthwhile to pointing out that current research on time delay of MRF dampers have been focused on the control response (force) respect to the given current input rather than the control response (force) respect to the command signal. However, the actual responses time in a control system should be the physical response of a control device respect tithe command signal rather than the intermediate signal (such as current).In addition, although there have been a certain amount of publications on the real-time control implementations of MRE device, critical issues such as the response time of MRE device, has not yet been properly addressed. For example, a main difference in MRF devices and MRE devices is that the design of MRE devices usually adopts larger electromagnetic coils thus complex design of driving electronics is inevitably required for the device. The response time effects due to such design features of MRE devices have not yet to be investigated.

In this paper, a new testing method is proposed to determine the response time of stiffnessvariable MRE device for real-time control implementation. Using such setup, the response time of a MRE isolator, i.e. a multilayer MRE base isolator [7] with large solenoid coil, is evaluated experimentally. Analysis on the response time of the generated force validates that the inductance of the coil and the electrical impedance have major contribution to the overall response time of the device. Therefore, several approaches are applied to reduce the impact of the coil inductance and electric impedance. The overall response time of the MRE base isolator has been successfully reduced in both rising and falling edges. The feasibility of its implementation for real-time control is validated through a case study.

\section{Experiment set-up}


This study is conducted on a multilayer MRE base isolator with a single large coil (electric resistance of $42.3 \Omega$ ) to provide strong magnetic field. Detailed design of the isolator can be found in the reference [7]. The experimental setup is shown in Figure 1. MRE isolator is fixed on the shake table which undertakes designated motion during testing. A load cell is installed in-between the MRE isolator and the reference wall for shear force measurement. dSPACE system is used here for dual-purposes: 1) to apply step control signal which inputs to driving electronics and then the electromagnetic coil; and 2) to synchronize the force and current transient responses.

To achieve a fast response time of the current, a PWM servo current source regulated by PI controller is designed, shown in Figure 2. The frequency of PWM signal is set as $1 \mathrm{kHz}$ so an IGBT (insulated gate bipolar transistor) electronic switch is adopted to deliver the high frequency switching logic. The circuit description of the IGBT drive is depicted in Figure 7. The PI controlled PWM circuit can drive the isolator with full capacity to acquire swift response curve in the beginning (with little overshooting) and regulate the applied voltage to maintain a stable current once the current in the coil reaches the desired value.

As mentioned earlier, different from MRF dampers, the MRE isolators are considered as stiffness-variable devices in which the force generated are mainly dependent on the deformation. Hence, constant displacement should be maintained to evaluate the transient force response of the isolator subjected to step change of the control command. Involvement of any other form, such as velocity, will distort the shear force due to change of the displacement and hence should not be introduced. The displacement signal shown in Figure 3 with $2 \mathrm{~mm}, 4 \mathrm{~mm}$ and $6 \mathrm{~mm}$ is selected in the test.

To observe both the rise and fall edges of the response, a square-shape control signal with a period of 10 s was applied to the MRE isolator at each displacement stage. The peak control signal corresponds to an applied current of $3 \mathrm{~A}$ by amplification of the driving electronics. 
The current and force responses of the MRE isolator applied with constant displacement of $4 \mathrm{~mm}$ are illustrated in Figure 4. The response time is measured from initial stage to $95 \%$ of the final transition for both current and force responses (Table 1). It is worthwhile to highlight that the response time measured in this research is the time lag between force response and the control command, rather than force response respect to applied current usually adopted in existing research publications.

MRE isolator used here has large coil with 2900 winding turns and resistance of $42.3 \Omega$. The force response times (with respect to control command) of the rising and falling edges are $421 \mathrm{~ms}$ and $402 \mathrm{~ms}$, respectively. As observed, the majority of the response time, about $73 \%$, is consumed during the conversion from control command to the applied current. There is a relatively small time lag (around $110 \mathrm{~ms}$ ) between the current and force responses, which indicates the rapid response of MRE material when subjected to the changing magnetic field. Comparison between two driving electric circuits, i.e. with and without PWM servo, proves the effectiveness of the PWM servo source. The force response time of the setup without PWM servo source exceeds $1200 \mathrm{~ms}$. To summarize, MRE isolator's time delay is mainly caused by the building up of the current due to massive inductance of the solenoid and the driving electronics which converts the control command (usually 0-5V) into the desired current. Hence, the modifications in section 4 are on the techniques to realize fast current response.

\section{Response time definition}

Neglecting eddy currents in the steel components, the basic behavior of the electromagnetic coil can be modeled using a resistor and an inductor in series connection. The circuit diagram of the solenoid is illustrated in Figure 5. Therefore, the current flow in the coil can be written as:

$$
i(t)=I_{H}\left(1-e^{-\frac{t}{\tau}}\right)
$$




$$
i(t)=I_{\mathrm{H}} e^{-\frac{t}{L_{0} / R_{0}}}
$$

in which, $L_{0}$ and $R_{0}$ a are the inductance and resistance of the coil, respectively; $\tau=L_{0} / R_{0}$ is the time constant of this circuit. According to Equation (1) and (2), $t=3 \tau$ is the time required to accomplish $95 \%$ of transition change at both rise and fall edges, and thus is defined as the response time. The illustration of response time is displayed in Figure 6.The single coil used in the MRE base isolator has high inductance $(3.5 \mathrm{H})$, which leads to slow response of the device, shown in Table 1.

\section{Approaches}

In this section, two approaches are proposed to reduce the response time of the MRE base isolator, i.e. parallel coil configuration and field quenching design. The parallel coil configuration separates a single large coil into several smaller coils which reduces the coil inductance and maintains same level of magnetic field to the MRE materials. The field quenching design aims to improve the efficiency of the magnetic circuit by eliminating the magnetic field residence with a special design of the coil and feedback loop.

1) Parallel coil configuration

To reduce the inductance, the large coil used in the design is separated into several identical smaller coils which are connected in parallel. To achieve same level of magnetic flux density, the total turn number of the coils maintains as the same as the original coil.

When $n$ identical coils are in parallel connection and each coil is energized by a current $I_{H}$, equivalent resistance and inductance of the circuit can be now written as

$$
R=\frac{R_{0}}{n^{2}} \text { and } L=\frac{L_{0}}{n^{2}}
$$

To maintain $I_{H}$ in each coil, the total current of the circuit is $n I_{H}$. Consequently, the governing current of the parallel coils circuit can be given as

$$
i(t)=n I_{H}\left(1-e^{-\frac{t}{L / R}}\right)=n I_{H}\left(1-e^{-\frac{t}{\tau}}\right)
$$


As can be seen from the equation, the time $t_{0}$ to reach $I_{H}$ id is

$$
t_{0}=\tau \ln \left(1-\frac{1}{n}\right)^{-1}=\frac{L_{0}}{R_{0}} \ln \left(1-\frac{1}{n}\right)^{-1}
$$

Conducting Taylor Expansion in the term of $\frac{1}{n}$ on the formula, $t_{0}$ can be approximated as

$$
t_{0}=\frac{L_{0}}{R_{0}} \frac{1}{n}+\frac{L_{0}}{2 R_{0}} \frac{1}{n^{2}}-\frac{L_{0}}{3 R_{0}} \frac{1}{n^{3}}+\cdots
$$

When $n$ is large enough, retaining only the first term in the series can provide a reasonably accurate estimate fort $t_{0}$. For instance, when $n=8$, the second term of Equ (6) is a negligible item, about $6.9 \times 10^{-4}$. It is obvious that the time to reach a desired current $I_{H}$ and the number of divided coils can be reduced to $1 / n$ of the original if the large coil is replaced by a sequence of smaller coils connected in parallel configuration. In the new design, the large solenoid of the MRE isolator was divided into eight identical smaller coils. The schematic of the isolator with new set of coils is shown in Figure 8.

2) Magnetic field quenching configuration

Residual magnetic field is a challenge to create a fast falling edge during the transient period due to the hysteresis existing in steel plate, steel yoke and MRE materials of the isolator. To overcome this, a special arrangement of the coils is adopted to remove the residual magnetic field by create opposite magnetic field during falling period. In the proposed configuration, eight coils are divided into two sections, namely, energizing section (with six coils) and fieldquenching section (with two coils sitting at the top and bottom). In the PWM servo current source shown in Figure 2, during the rising period, only the energizing section is regulated by the PWM signal. When the command signal comes to a fall edge, the field-quenching section is energized with opposite current direction to remove residual magnetic field until feedback current reaches to the desired value. The logic function of the energizing section's PWM duty cycle $\alpha_{\text {energise }}$ and field-quenching section's PWM duty cycle $\alpha_{\text {quench }}$ can be written as

$$
\left\{\begin{array}{c}
\alpha_{\text {energise }}=\alpha, \alpha_{\text {quench }}=0,0 \leq \alpha \leq 0.5 \text {, rising } \\
\alpha_{\text {energise }}=0, \alpha_{\text {quench }}=1,-0.5 \leq \alpha<0, \text { falling }
\end{array}\right.
$$


In which, $\alpha$ is the duty cycle calculated by PI controller. It is noteworthy that, instead of current feedback, a Hall Effect sensor is adopted to feedback magnetic field applied to the MRE material to avoid corrupted current feedback signal caused by the cross coupling between the quench and energizing coils. The circuit description of magnetic field quenching configuration is shown in Figure 9. In Figure 9, two identical PWM servo current sources described in Figure 2 are employed to drive the energizing section and field-quenching section separately.

\section{Results and discussion}

1) On current and force responses

To demonstrate effects of the proposed configurations, the same current input adopted in the original response testing is applied to the MRE isolator with a constant displacement of $4 \mathrm{~mm}$. Three different coil configurations were experimentally compared, including the series, parallel and field-quenching configurations. The transient current responses under different configurations are shown in Figure 10.

It can be clearly observed that, at rise edge, the parallel configuration achieves a much shorter response time than the series configuration. The response time of the parallel configuration is approximately $132 \mathrm{~ms}$ while that of series configuration requires $540 \mathrm{~ms}$.Suchresult convincingly demonstrates that the response time of the parallel configuration can be reduced by $n$ times compared to the series scenario. However, it can also be observed that the parallel configuration still receives considerable response time at fall edge, around $700 \mathrm{~ms}$, which is even slower than the series configuration, i.e. $580 \mathrm{~ms}$. The reason underlies the phenomena is that changing the connection method cannot cancel the effect of long decay time of the magnetic field due to magnetic residence. The field-quenching configuration, however, attained excellent response time reduction effect in both rising and falling edges. The rise time is approximately $44 \mathrm{~ms}$ while the fall time is about $40 \mathrm{~ms}$. The fluctuation of the current 
response in field-quenching configuration is due to the interaction of the inductance and the inter-winding capacitance. In both rising and falling periods, the current fluctuation is around \pm 0.3 A which also dampens down very quickly.

The force response under field-quenching arrangement was tested with the same control command excitation under three constant displacements. The response times of current and force responses under three displacements are revealed in Figure 11. As observed, the influence of displacement on either current or force response is not significant. Meanwhile, under any displacement, the current response has a smaller fall time than rise time, which proves the effectiveness of the field-quenching configuration.

The current and force responses under $4 \mathrm{~mm}$ displacement are displayed in Figure 12 and Table 2. It can be clearly seen from Figure 12 that the force response is as rapid as current response at rise edge which again indicates the fast response of the MRE material. The final force rise time and fall time are $52 \mathrm{~ms}$ and $48 \mathrm{~ms}$, respectively. Compared to the original response time, both current rise and fall time have been reduced by $87 \%$ and force rise and fall time have been reduced by $88 \%$. Fluctuation of the current does not create large variation in the force rising transient. In the falling transient, the fluctuation of the force is caused by the mechanical connection due to sudden loss of the system stiffness rather than the fluctuating current.

2) Performance evaluation for real-time control implementation

Further evaluation was conducted to verify the feasibility of MRE isolators for real-time control implementation from structural control point of view. Seismic test of a smart base isolation system comprising a 3-storey building model and MRE base isolators is used with a simple on-off control algorithm for such purpose. Since the shear force generated by the MRE isolator cannot be directly measured, the current responses under control command are used with comparison between field-quenching configuration and parallel configuration in 
Figure 13. With the field-quenching configuration, the MRE isolator is able to simultaneously follow the control signal in both rise and fall transients. MRE isolator with parallel configuration behaves well in the rise time with struggles to maintain constant current. However, in the fall time, considerable time delay is observed during the fall time which inevitably downgrades the control performance.

It is discovered that the control performance is significantly deteriorated when the ratio between real time delay and critical time delay is greater than 0.6 , in which critical time delay represents the time delay rendering the controlled structure to be unstable [17]. For a SDOF system, the critical time delay is approximately 0.25 of the natural period of uncontrolled system. Although critical time delay of MDOF system is much more complicated than that of a SDOF system, the aforementioned approximation is still instructive when evaluating the effect of time delay to the controlled structure. Hence, the critical time delay and thus allowable time delay of the control system can be even smaller if higher modes of the MDOF system are also controlled [18]. In the test, the fundamental period of the 3-storey building model is $0.53 \mathrm{~s}$. Therefore, the critical time delay of the system should be less than $132 \mathrm{~ms}$ and allowable time delay of the system should be less than $79 \mathrm{~ms}$ to avoid jeopardizing the stability and performance of the system. With a fast response time, i.e. $52 \mathrm{~ms}$ and $48 \mathrm{~ms}$ in rise and fall time, the proposed MRE isolator has convincing capability for real-time control implementation.

\section{Conclusion}

This paper reported an investigation for improving response time of a MRE isolator for realtime control implementation. A testing method was firstly introduced to examine the response time of MRE isolators. Due to inherent large coil design in the MRE devices, there is significant delay in response time resulted from the inductance of the coil and the electrical impedance which needs to be resolved in order to achieve real-time control. From the 
experiment it was observed that the delay in response time is mainly due to the delay of the coil current response. To resolve this issue, two approached were introduced to reduce the response time of MRE isolator. The first approach is to arrange the large coil with several identical coils (for example, $n$ parallel coil). With the proposed design, the response time can be reduced to be $1 / \mathrm{n}$ of the original response time. To eliminate the influence of the residual magnetic field, a field quenching configuration was design by driving top and bottom coil with opposite current during falling time. It was found out that the modified design can reduce the force response time from $421 \mathrm{~ms}$ to $52 \mathrm{~ms}$ in the rising and from $402 \mathrm{~ms}$ to $48 \mathrm{~ms}$ in the falling, respectively. An experimental evaluation of real-time control of a MRE isolator with the proposed time response reduction approaches confirmed that this isolator is adequate for real-time control implementation for seismic protection.

\section{References}

[1] Li Y C, Li J C, Li W H and Du H P2014 A state-of-the-art review on magnetorheological elastomer devices Smart Materials and Structures23 123001

[2] Li W H, and Zhang X Z 2010 A study of the magnetorheological effect of bimodal particle based magnetorheological elastomers Smart Materials and Structures19035002

[3] LiaoG J, Gong X L, Xuan S H, Kang C J and Zong L H 2012 Development of a real-time tunable stiffness and damping vibration isolator based on magnetorheological elastomer Journal of Intelligent Material Systems and Structures 23 25-33

[4] Du H P, Li W H, and Zhang N 2011 Semi-active variable stiffness vibration control of vehicle seat suspension using an MR elastomer isolator. Smart materials and structures, 20(10), 105003

[5] Behrooz M, Wang X, and Gordaninejad F2014 Modeling of a new semi-active/passive magnetorheological elastomer isolator. Smart Materials and Structures 23045013 
[6] Shi HF, Yu M, Zhu, M, Fu J, Choi SB and Xing ZW2016 An investigation of the dynamic behaviors of an MRE isolator subjected to constant and alternating currents Smart Materials and Structures $\mathbf{2 5 0 7 7 0 0 2}$

[7] Li Y C, Li J C, Tian T F and Li W H 2013 A highly adjustable magnetorheological elastomer base isolator for applications of real-time adaptive control Smart Materials and Structures $\mathbf{2 2}$ 095020

[8] Du H P and Zhang N2008 Hळ control for buildings with time delay in control via linear matrix inequalities and genetic algorithms Engineering Structures3081-92.

[9] Karimi HR2011 Robust delay-dependent control of uncertain time-delay systems with mixed neutral, discrete, and distributed time-delays and Markovian switching parameters IEEE Transactions on Circuits and Systems I: Regular Papers581910-23

[10] Abdel-Rohman M, 1987 Time-delay effects on actively damped structures Journal of engineering mechanics1131709-19

[11] Van de Beek JJ, Sandell M and Borjesson PO 1997 ML estimation of time and frequency offset in OFDM systems IEEE transactions on signal processing 451800-5

[12] Weiss KD, Duclos TG, Carlson JD, Chrzan MJ and Margida AJ1993 High strength magnetoand electro-rheological fluids Society of Automotive Engineers Technical Paper Series, Warrendale 932451 1-6

[13] Choi YT and Wereley NM 2002 Comparative analysis of the time response of electrorheological and magnetorheological dampers using nondimensional parameters Journal of Intelligent Material Systems and Structures13443-52

[14] Koo JH, Goncalves FD and Ahmadian M 2006 A comprehensive analysis of the response time of MR dampers Smart materials and structures $\mathbf{1 5 3 5 1}$

[15] Strecker Z, Roupec J, Mazurek I, Machacek O, Kubik M and Klapka M2015 Design of magnetorheological damper with short time response Journal of Intelligent Material Systems and Structures $\mathbf{2 6} 1951-8$

[16] Yang G, Spencer BF, Carlson JD, Sain MK2002 Large-scale MR fluid dampers: modeling and dynamic performance considerations Engineering Structures24309-23 
[17] Agrawal AK, Fujino Y and Bhartia BK1993 Instability due to time delay and its compensation in active control of structures Earthquake engineering \& structural dynamics 22211-24

[18] Agrawal AK and Yang JN1997 Effect of fixed time delay on stability and performance of actively controlled civil engineering structures Earthquake Engineering \& Structural Dynamics 261169-85 


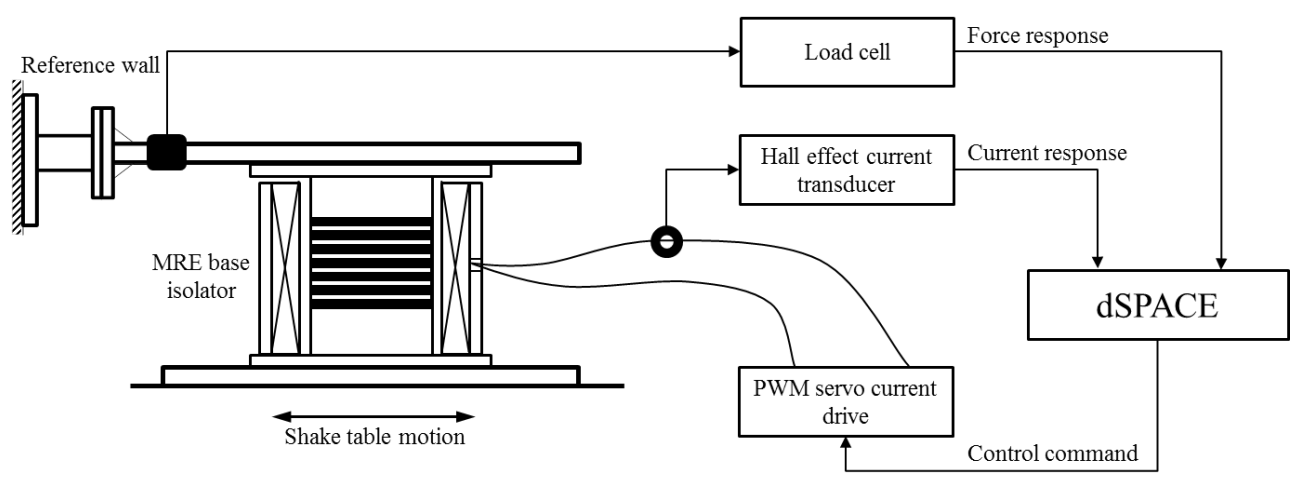

Figure 1 Experimental setup of force response testing 


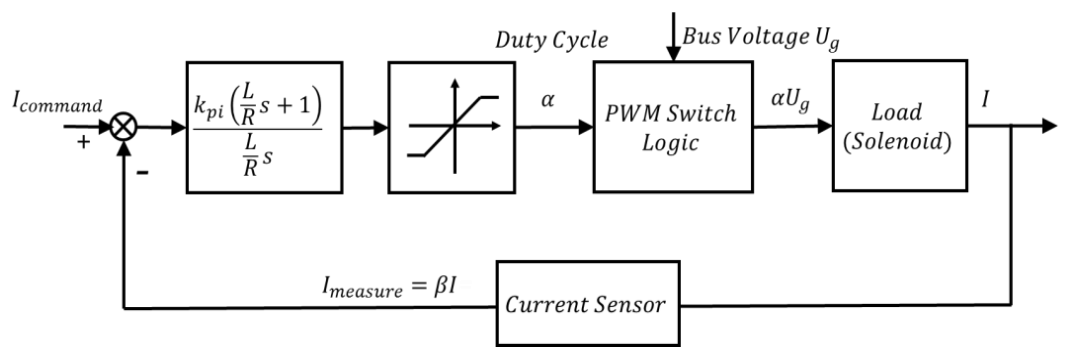

Figure 2 Flow chart of PWM servo current source 


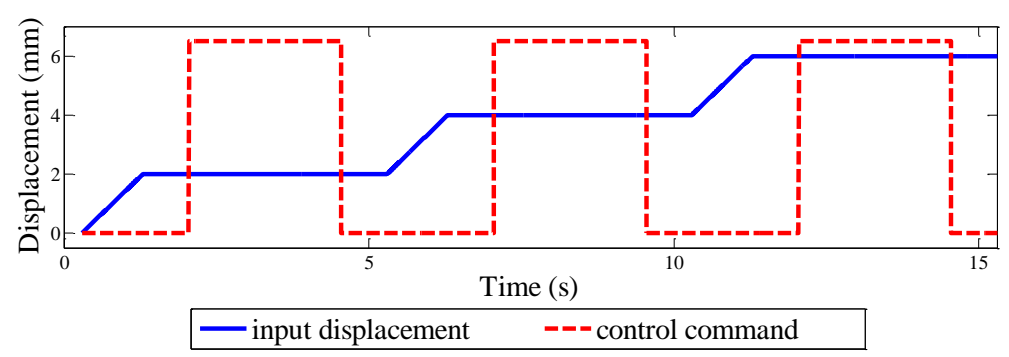

Figure 3 Illustration of displacement and current inputs 

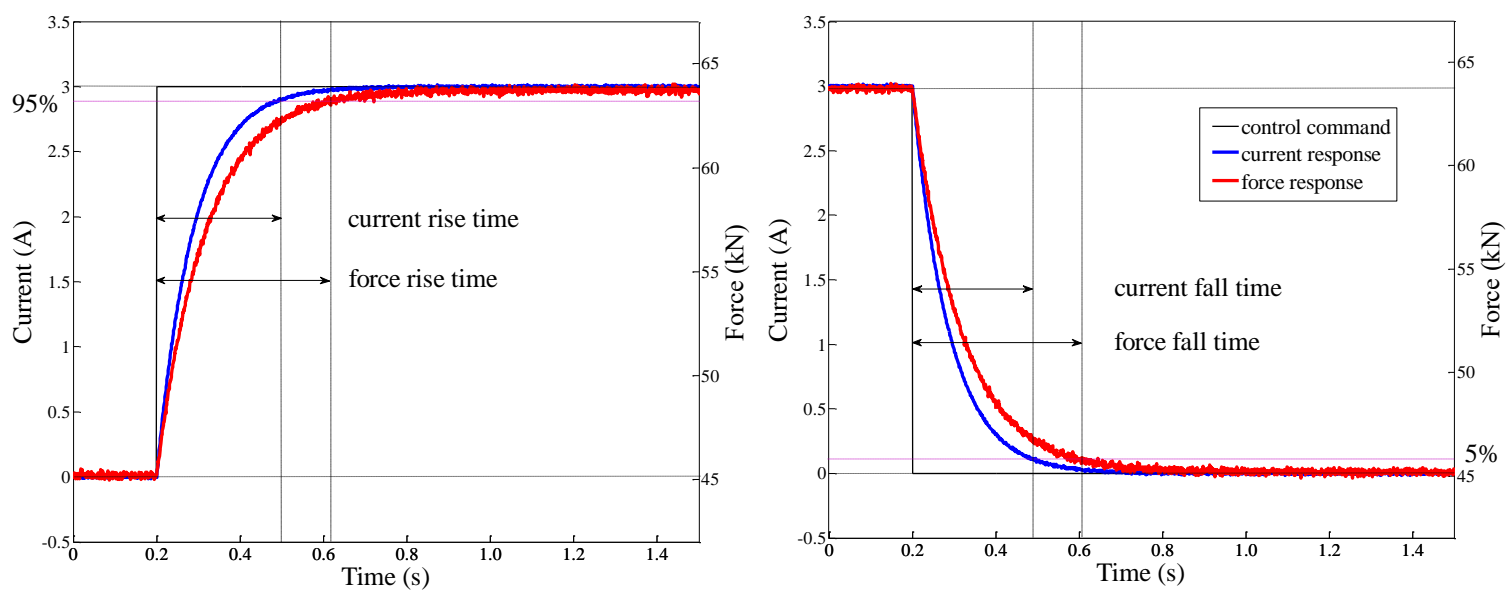

Figure 4 Original current and force response of MRE isolator 


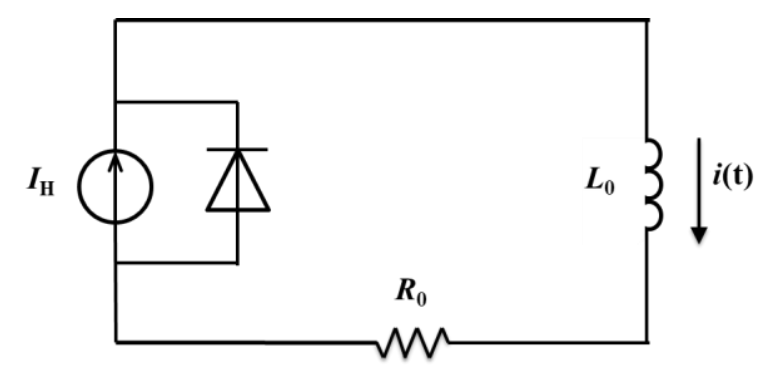

Figure 5 Circuit diagram of the solenoid with current sources 

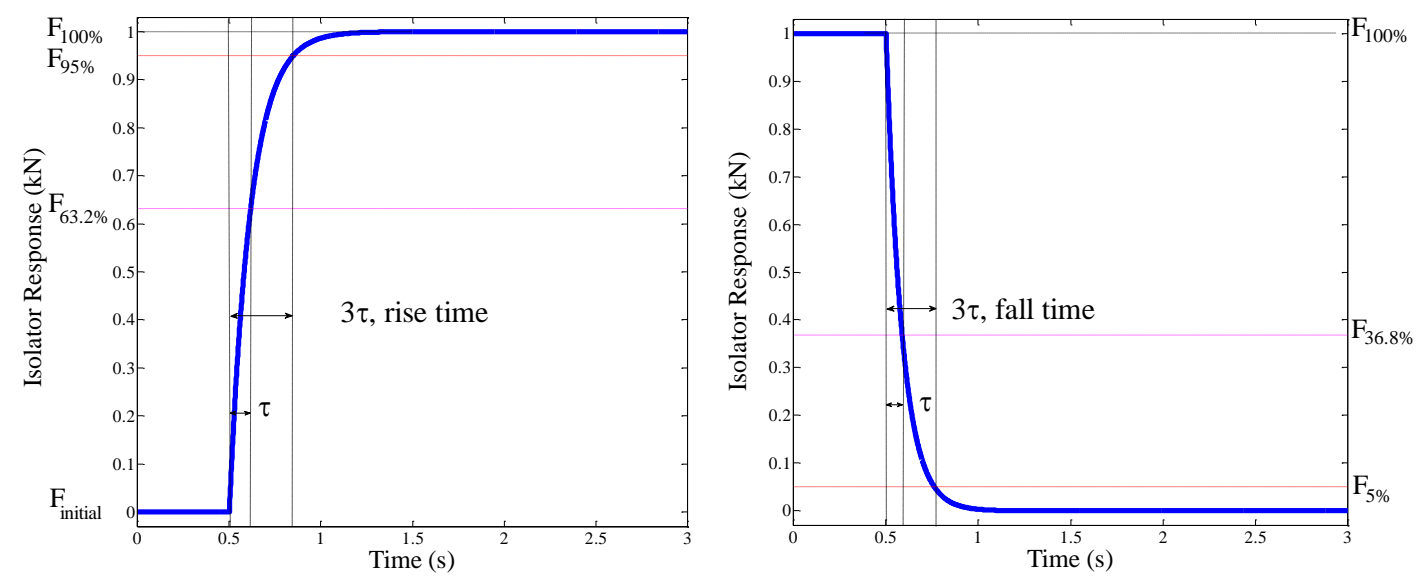

Figure 6 Definition of response time at rise edge and fall edge 


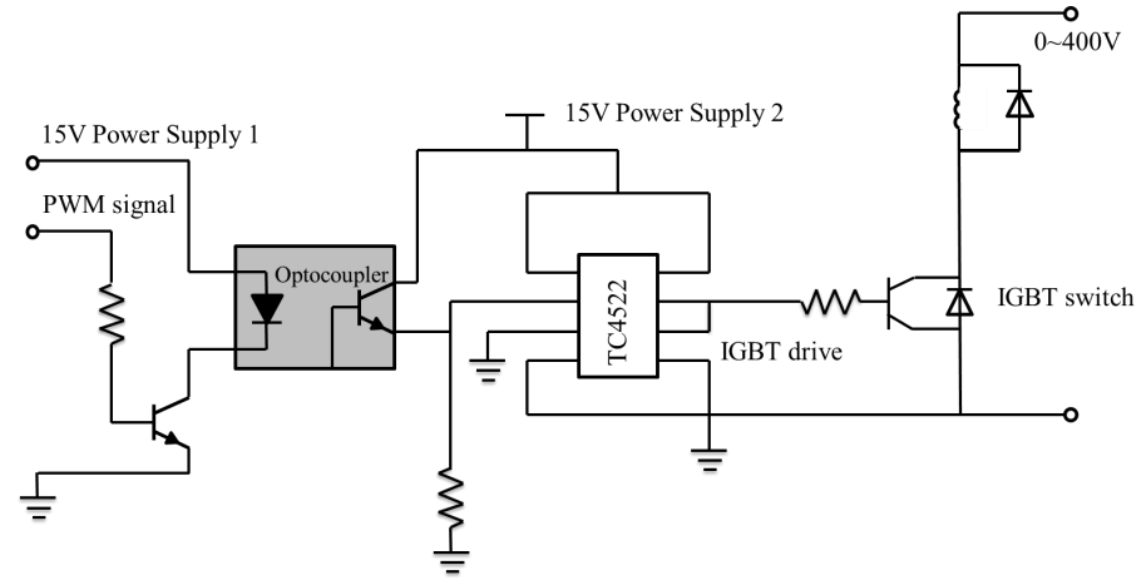

Figure 7 Circuit description of isolated IGBT drive driven by PWM signal 


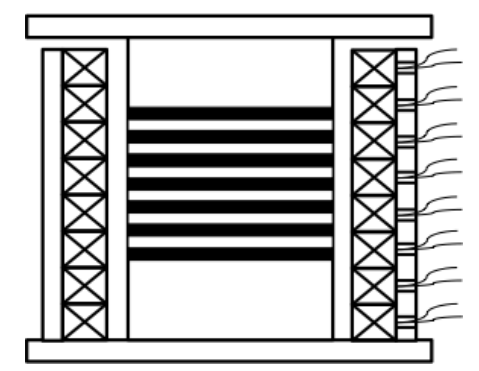

Figure 8 Schematic diagram of MRE isolator with multi coils 


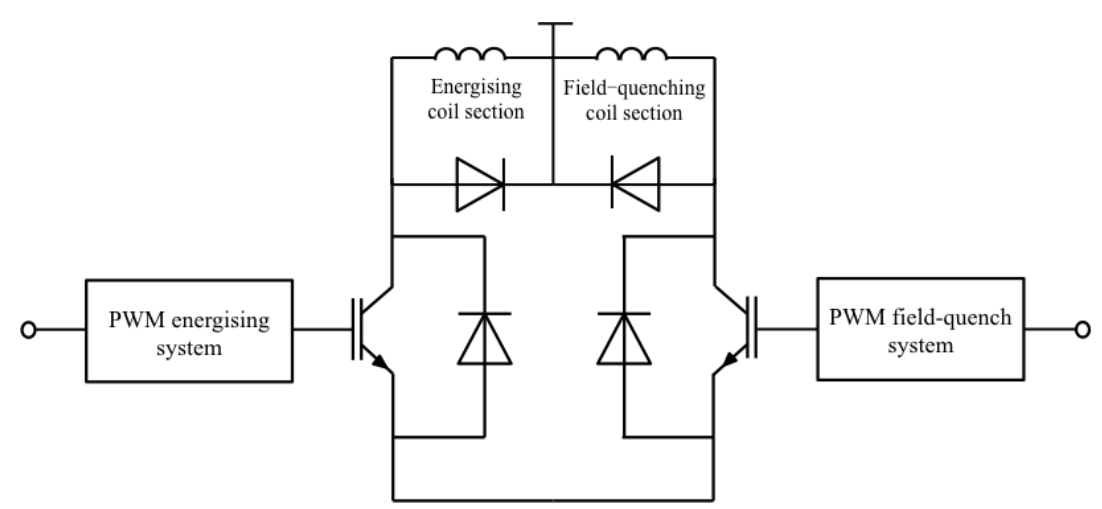

Figure 9 Circuit description of split coil system 

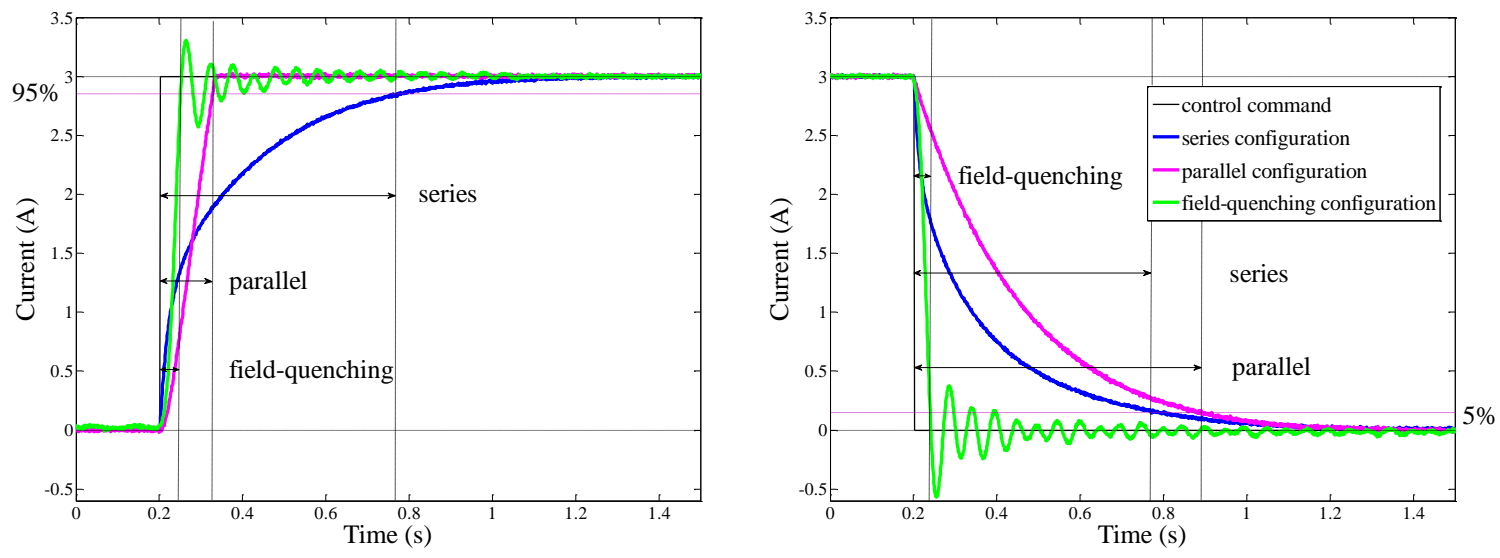

Figure 10 Current response curves under different coil configurations 


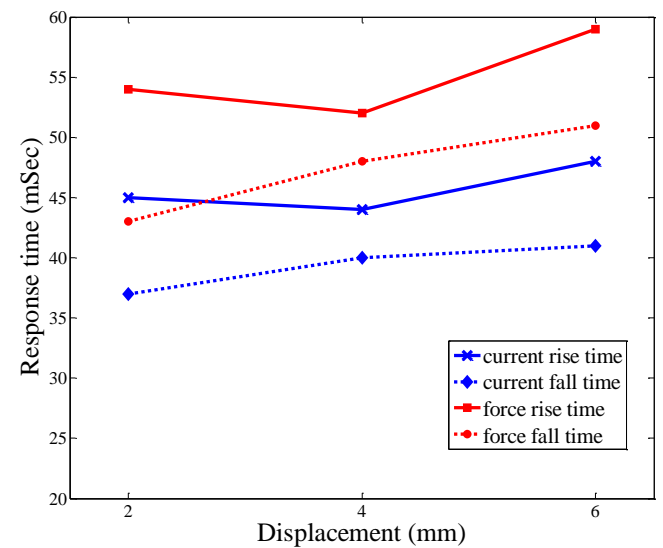

Figure 11 Current and force response time under different displacements 

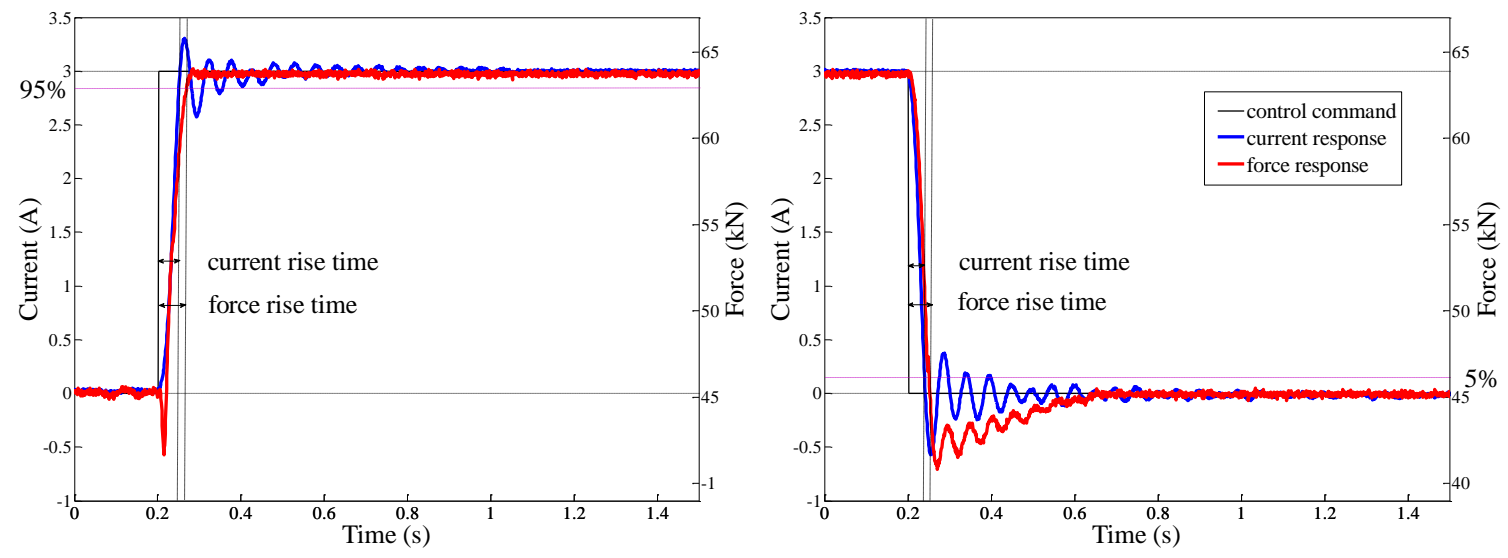

Figure 12 Final current and force responses with split coils configuration 

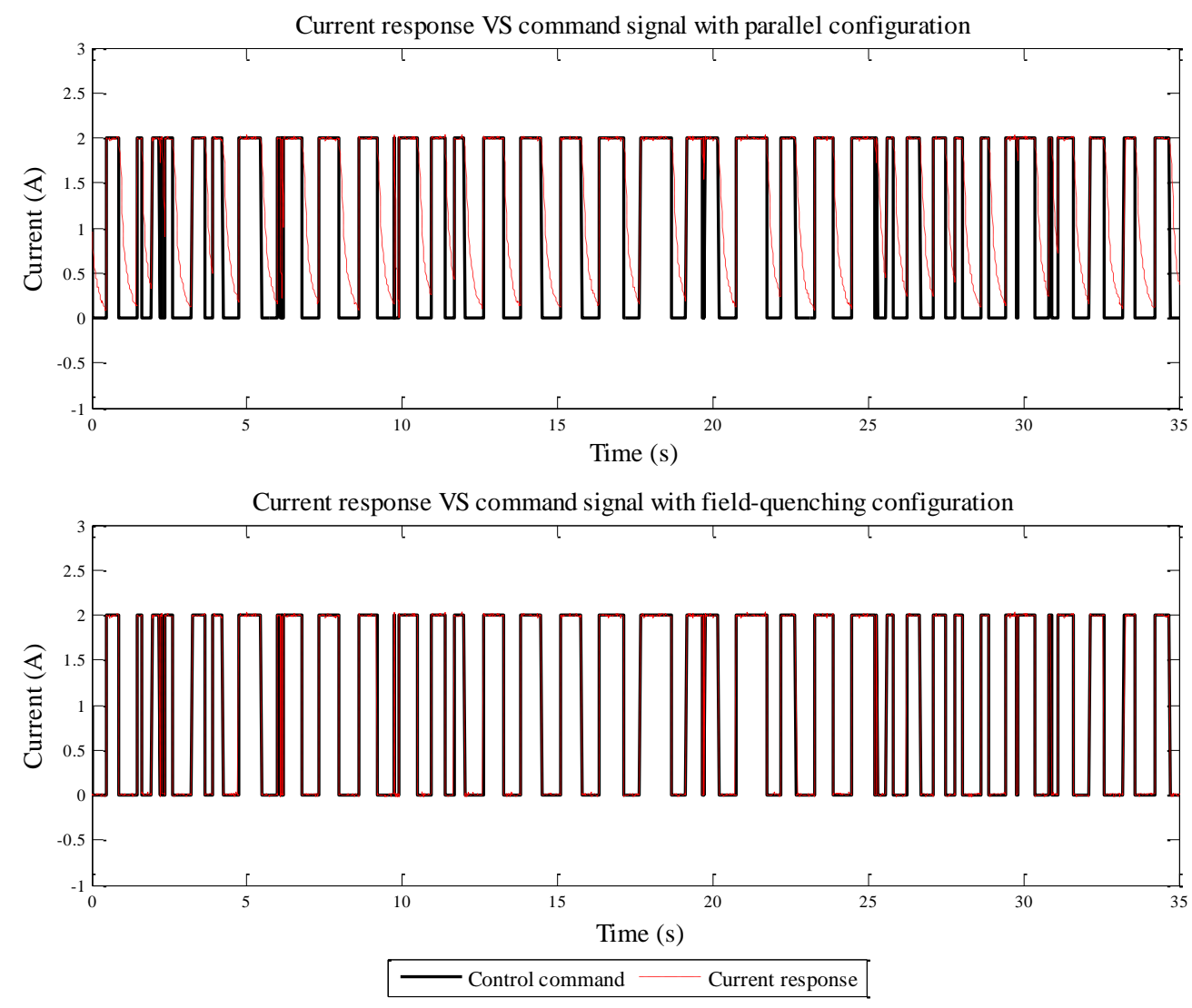

Figure 13 Current responses under split coil and parallel configurations when the MRE isolator energised with practical control command 
Table 1Original current and force response time (4mm displacement)

\begin{tabular}{ccccc}
\hline & \multicolumn{2}{c}{ Current response } & \multicolumn{2}{c}{ Force response } \\
\hline $\begin{array}{c}\text { Response time } \\
(\mathrm{mSec})\end{array}$ & Rise edge & Fall edge & Rise edge & Fall edge \\
\cline { 2 - 5 } & 308 & 296 & 421 & 402 \\
\hline
\end{tabular}


Table 2Final current and force response time (4mm displacement, field-quenching configuration)

\begin{tabular}{ccccc}
\hline & \multicolumn{2}{c}{ Current response } & \multicolumn{2}{c}{ Force response } \\
\hline $\begin{array}{c}\text { Response time } \\
(\mathrm{mSec})\end{array}$ & Rise edge & Fall edge & Rise edge & Fall edge \\
\cline { 2 - 5 } & 44 & 40 & 52 & 48 \\
\hline
\end{tabular}

